\title{
Resistance Exercise Machine within Lower Body Negative Pressure for Counteracting Effects of Microgravity
}

\author{
Christine M. Dailey ${ }^{4}$,Charles Reinholtz ${ }^{1}$, Thais Russomano ${ }^{2}$, Michael Schuette $^{3}$, Rafael Baptista ${ }^{2}$, and \\ Rodrigo Cambraia ${ }^{2}$
}

${ }^{1}$ Embry-Riddle Aeronautical University, Daytona Beach, FL; ${ }^{2}$ Microgravity Centre, Brazil; ${ }^{3}$ Sotera Defense Solutions, Inc., Crofton, MD; ${ }^{4}$ Naval Research Laboratory, Washington, DC

\begin{abstract}
Physiological deconditioning is a critical problem in space, especially during long-term missions. Resistance exercise, coupled with lower body negative pressure (LBNP), has been shown to be effective in counteracting some of the deconditioning related problems. This paper describes the development of a compact and effective resistance exercise machine that works within an existing environmentally controlled LBNP Box, and is designed to simulate both exercise and sitting, to decrease microgravityinduced deconditioning by simulating physiological and biomechanical features of upright exercise and daily activities. Theoretical calculations are carried out to determine whether kinematics, musculoskeletal loadings, and metabolic rate during supine exercise within the existing LBNP Box are similar to those of an upright posture in Earth gravity (1G).
\end{abstract}

Key words: Lower Body Negative Pressure; Space

Exercise; Microgravity; Effects Of

Microgravity; Resistance

Exercise; Osteoporosis; Weightlessness;

Cardiovascular Deconditioning; Exercise

Countermeasure

Correspondence to: Christine M. Dailey

Mechanical Engineer

Naval Research Laboratory

Washington, DC, USA

Telephone: 202-404-1906

E-mail: Christine.dailey@nrl.navy.mil
Preliminary results show subjects that use the resistance machine presented in this paper will be able to elicit loads comparable to exercise on Earth, since the ground reaction forces are greater than their body weight (BW). The largest singleleg forces during resistance exercise are $1.16 \mathrm{BW}$ (232 lbs) during supine position when $\gamma$, the angle between the horizontal and the ground pivot on the right side of the mechanism, equals 187 degrees and minimal at $0.68 \mathrm{BW}(136 \mathrm{lbs})$ when $\gamma$ equals 177 degrees. At the lowest setting of the machine, peak resistance of the foot pedal during the outward stroke is $196 \mathrm{lbf}$. This force, added to the force due to the $50 \mathrm{mmHg}$ of negative differential pressure, gives a total force of $400 \mathrm{lb}$, which is $2 \mathrm{BW}$.

The results suggest that this machine can be used to collect and establish a database under both terrestrial conditions and microgravity environments, such as the International Space Station (ISS), to enhance medical researchers' understanding of how LBNP paired with exercise impacts osteoporosis, orthostatic intolerance, and cardiovascular health.

\section{INTRODUCTION}

Gravity has had an integral effect on the development of life on Earth over millions of years and has shaped the anatomy and physiology of human beings. Exposure to microgravity has been shown to affect the body, causing it to undergo a reduction in heart size (Dorfman et al., 2007) and blood volume (Watenpaugh, 2001), 
central nervous head and spine impaired balance control (Mulavara et al., 2010), changes in nervous system sensitivity, specifically the vestibular system (Benson, 2002), decreases in bone and muscle mass (Morgan et al., 2012), and reduction of the immune function (Martinelli et al., 2009). Astronauts in space during short or long-term missions have demonstrated these physiological changes, known as space deconditioning, which may lead to undesirable health consequences and to operational difficulties, especially in emergency situations during the return to Earth (Hughson et al., 2012; Baqai et al., 2009).

In the future, it is expected that greater numbers of astronauts will work and live in lowgravity environments, and the need to understand the in-flight and post-flight consequences of microgravity exposure will become more significant. The physiological adaptations to weightlessness seem to be better tolerated by the astronauts than the changes suffered after they return to Earth. In space, the mechanical unloading affects the musculoskeletal system (Smith et al., 1998), causing significant atrophy, especially affecting the anti-gravitational group of muscles. It has been demonstrated that the vastus meadilias, vastus lateralis, gastrocnemius medialis, and tibialis anterior presented an important degree of atrophy after five weeks of a ground-based microgravity simulation (Berg et al., 2007). During actual exposure to microgravity, the calf muscle can decrease $13 \%$ after five months on board the ISS (Shackelford et al., 2004). The decrease in bone mass is also of great concern to space physiologists and physicians, as the normal processes of bone formation and resorption are disturbed, favoring a loss of bone tissue (Caillot-Augusseau et al., 1998). This process begins after the introduction into microgravity and can range between $1 \%$ and $2 \%$ of bone mass loss per month, affecting astronaut's health during the return to Earth (Lang et al., 2004). One of the first responses to spaceflight is the shift of blood and body fluids towards the upper body, with subsequent adaptations occurring over a few days to lower overall blood volume through activation of several mechanisms (Charles and Lathers, 1991; Thornton et al., 1977). It is upon return to Earth that the cardiovascular deconditioning raises concerns by producing significant orthostatic intolerance and decreasing aerobic performance (Convertino and
Cooke, 2005).

Many different types of countermeasures have been developed (ranging from specific diets to heavy exercise protocols) that must be performed periodically by the astronauts during a space mission. However, it is believed that a very efficient way to counterbalance space deconditioning is by improving the exercise countermeasures protocols, devices, and systems aiming to provide the terrestrial gravitational stress to the osteomuscular and cardiovascular systems. Among the countermeasures currently under testing, resistance exercise training regimen in space seems to be the most complete because it prevents increased bone resorption (Shackelford et al., 2004; Trappe et al., 2009), muscle loss, and cardiovascular deconditioning. However, the effectiveness of exercise protocols (aerobic and resistance exercise) and equipment (treadmill, cycloergometer, and Resistive Exercise Device (RED)) for astronauts in space is unresolved and still under discussion. Studies indicate that all exercise in space to date has lacked sufficient mechanical and physiological loads to maintain preflight musculoskeletal mass, strength, and aerobic capacity (Davis and Cavanaugh, 1993; Gazenko et al., 1976; Vorobyev et al., 1976; Watenpaugh and Hargens, 1996). Recently, researchers have been pairing exercise with LBNP. The LBNP Box is a sealed chamber into which the human subject is partially inserted. A seal near the waist allows a vacuum to be applied to the chamber, thus creating a lower relative pressure on the subject's lower body. This lower pressure helps pull bodily fluids toward the feet (Russomano et al., 2005).

Combining the resistive force from exercise and a uniformed pressure distribution to the lower extremities has shown to be an efficient solution for counteracting microgravity-induced deconditioning during terrestrial testing. A study of the addition of a treadmill to an LBNP Box has demonstrated that it is able to simulate physiological and biomechanical features of upright exercise (Boda et al., 2000). However, its mechanical design lacks mobility and is both large and heavy, making it unsuitable for spaceflight.

The research presented in this paper offers a new method that combines both, endurance and resistance exercise protocols. Preliminary data from our early test phases using gas analysis and electromyography showed that the physical effort required by the subject is considered enough to 
maintain cardiovascular and skeletal muscle fitness. The purpose was to design a lightweight, compactable exercise machine combined with a collapsible chair that could be easily integrated into a smaller, existing LBNP Box. The exercise device is to offer a constant load path to maintain compressive loads on the musculoskeletal system and aid to the human body as much as possible. The human body is a highly nonlinear mechanical device from the standpoint of generating forces over a given cycle of motion. The human strength curve for the leg-press exercise shows that the maximum force a user can produce at each point in the outward cycle gradually increases. Not surprisingly, we are able to generate far more force at the extreme position (when the knee joint is at full extension) than when the knee is sharply bent (Schulz et al., 2012). Mechanical work and effort in the muscles will be nearly optimized in relation to the muscle mechanics when the resistance provided by a machine most nearly matches this trend (Zwart et al., 2007). The resistance curve should match the human strength curve for optimal efficiency in strengthening muscle and stressing bone. Although the strength curve varies from user to user, the general shape of the curve is approximately maintained.

Our goal in designing this system was to match the resistance provided by the machine with the human strength curve in a leg press exercise. This requires an adjustable level of resistance that will lead to a vertical shift in magnitude of resistance while keeping the general shape of the curve to accommodate each user. In this paper the alternative system is referred to as a multi-platform. The multi-platform is to be a compact system that offers a constant load path throughout the cycle, and is to conform to the most natural movement of the human body as possible. The design was driven by both the dimensions of an existing LBNP Box and by the average size astronaut. Averaging the size and weight of astronauts (NASA, 2000) allowed for an initial range of resistance the multi-platform would impose on the user to simulate forces equal to one or more of their body weight (BW).

\section{METHOD}

A 3-D SolidWorks model of the multiplatform is shown in Figure 1. The integrated system, the multi-platform, and the existing LBNP Box, is shown in Figure 2 where the browncolored links simulate the human legs and feet. The green and red tubes represent cooling ducts that provide an environmentally controlled atmosphere. The system will stress the lower extremities of the human body by providing both a resistance force due to the exercise machine, and a pressure force caused by the LBNP Box. It is believed that these forces might help to counteract some of the undesirable effects of microgravity.

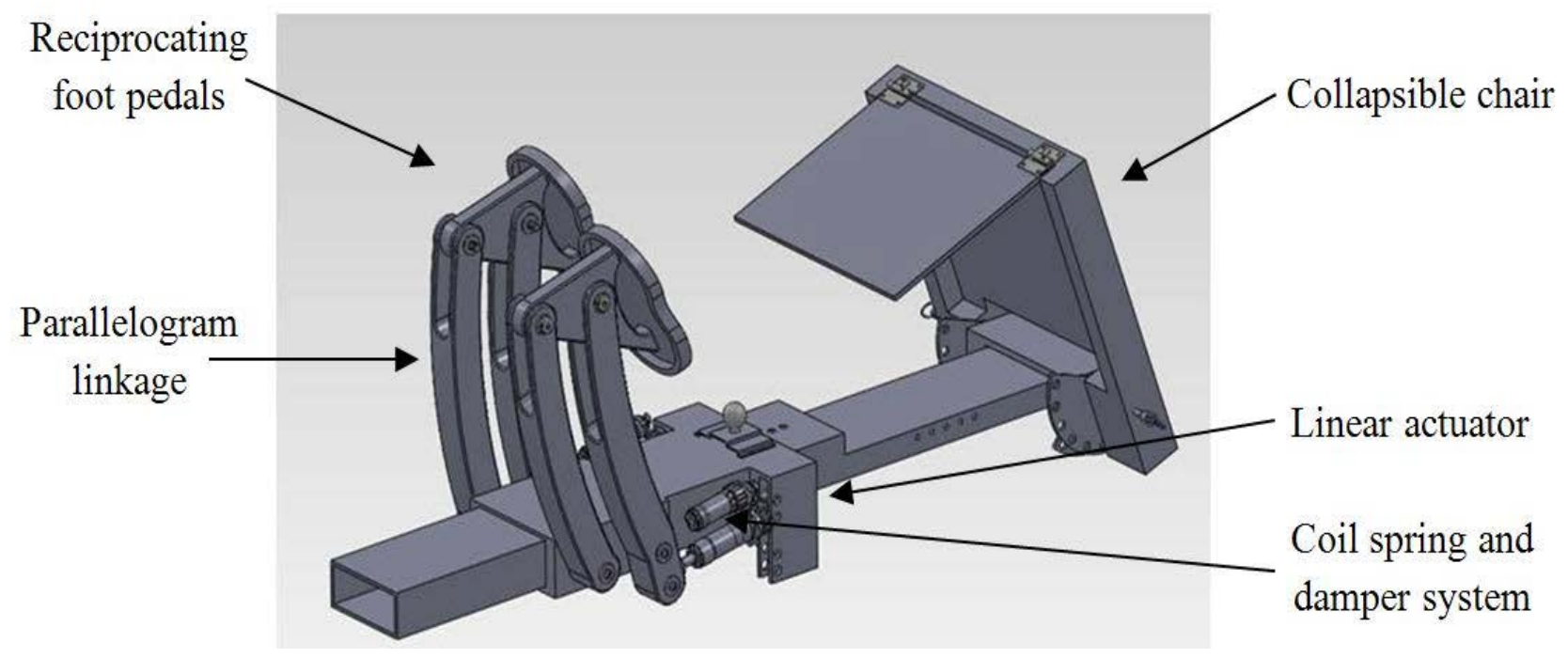

Figure 1. 3-D model of the multi-platform. 


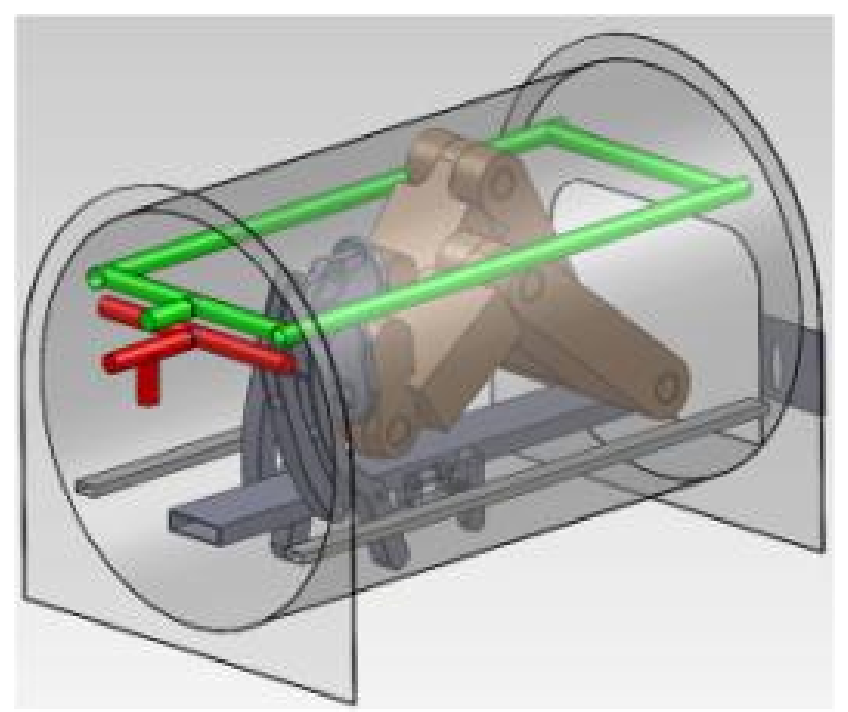

Figure 2. The multi-platform paired with an existing environmentally controlled LBNP Box.

Table 1. Specifications for the contributing links in the Moments of Inertia (MOI) equations discussed in section Kinematics.

\begin{tabular}{|c|c|c|c|c|c|}
\hline \multicolumn{6}{|c|}{ Specifications } \\
\hline Link & Height (in) & Weigh (in) & $\begin{array}{l}\text { Depth } \\
\text { (in) }\end{array}$ & Mass (lbs) & Moment of Inertia \\
\hline & 2.48 & 2.06 & 1.97 & 0.87 & 3.13 \\
\hline & 16.50 & 2.06 & 1.97 & 13.2 & 1727.93 \\
\hline & 16.50 & 2.06 & 1.97 & 13.2 & 1727.93 \\
\hline & & & & $\begin{array}{c}\text { Sum of MOI } \\
\text { (kg*sq.in) }\end{array}$ & 3459 \\
\hline
\end{tabular}

Table 2. Spring specifications used in the resistance equations discussed in section Kinematics.

\begin{tabular}{|l|l|}
\hline \multicolumn{2}{|l|}{ Spring Properties } \\
\hline Stroke length $\quad$ (in) & 3 \\
\hline Eye to eye length $\quad$ (in) & 12.51 \\
\hline Spring rate $\quad$ (k) & 650 \\
\hline
\end{tabular}

Parallelogram Linkage in Conjunction with Coil Spring and Damper System

Kinematics

Classic techniques in kinematics were used to design and optimize the geometry and resistance which would produce desirable motion and force properties (Tidwell et al., 1996). As shown in Figure 3, if a force is applied by the user to the foot pedal, the parallelogram linkage will guide the foot pedal along a circular-arc path at a fixed angle relative to the frame of the machine. This is 


\section{Resistance Exercise Machine within LBNP Countermeasure}

important for maintaining a generally perpendicular relationship between the lower leg and the foot. Applying forces in this manner to the musculoskeletal system is believed to be one of the most efficient ways to counteract osteoporosis (Gianoudis et al., 2012).

Referring to Figure 4, loop closure, Equation (1), and velocity loop, Equation (2), will yield the position, s, and velocity, s-dot, of the slider crank mechanism given the input position, $\theta$, and velocity, $\theta$-dot. Static resistance is dependent only on the value of $\theta$, which determines the compression of the spring and the geometry of the device. Dynamic resistance depends on the user's motion profile $(\theta-$ dot $)$.

$$
\begin{aligned}
& \text { Equation (1): } l_{o} \hat{\jmath}-l_{1}-l_{2} e^{j \theta}-s e^{j \gamma}=0 \\
& \text { Equation (2): } \hat{\jmath} l_{2} \dot{\theta} e^{j \theta}-\dot{s} e^{j \gamma}-\hat{\jmath} s \dot{\gamma} e^{j \gamma}=0
\end{aligned}
$$

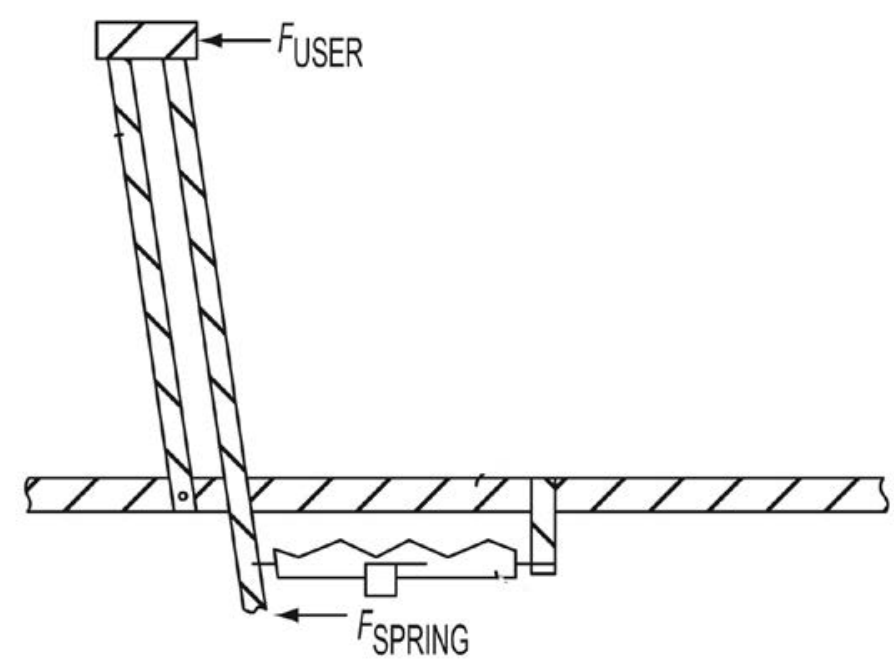

Figure 3. 2-D sketch of a four-bar parallelogram paired with a sliding crank mechanism. The sliding crank is a spring and damping system that offers a variable resistance.

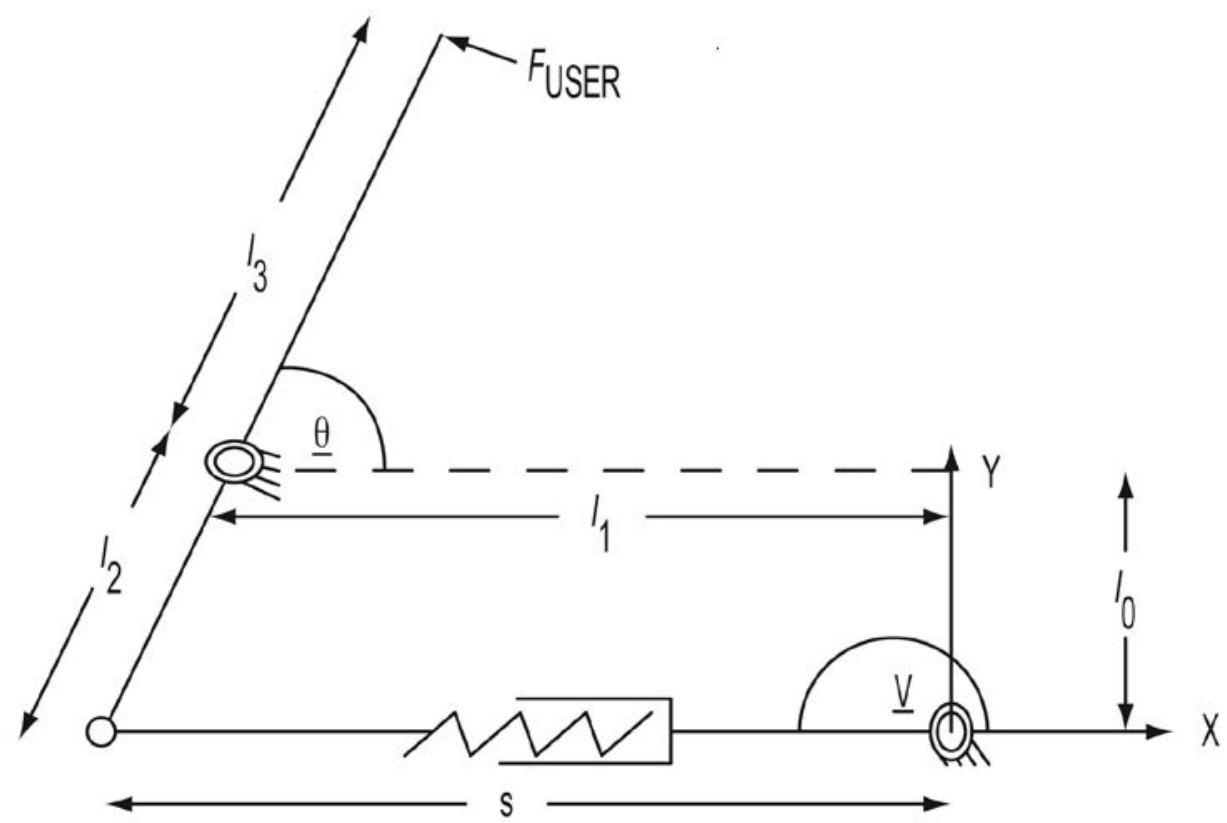

Figure 4. Kinematic diagram of the mechanism. 


\section{Resistance Exercise Machine within LBNP Countermeasure}

Two different motion profiles were used to calculate the inertia and damping force. The first profile had constant angular acceleration of the foot pedal link to start and end the motion cycle, and a period of constant velocity in between. The second motion profile was similar, but with no constant velocity motion period separating the periods of positive and negative constant acceleration. An electrogoniometer was used as a method in confirming which assumed motion profile was most accurate. The meter was applied to the subject's left knee, centered directly over the rotational joint.

Once the position and velocity loop equations have been solved, virtual work can be used to find the resistive force, $\mathrm{F}_{\text {user }}$, as a function of position, $\theta$, from Equation (3).

$$
\text { Equation (3): } I^{*} \ddot{\theta} \dot{\theta}+F_{\text {user }} \dot{\theta} l_{3}+F_{\text {spring }} \dot{s}=0
$$

The inertial term in Equation (3), $I^{*} \ddot{\theta} \dot{\theta}$, is based on a position-dependent equivalent inertia approach, as described in Suh and Radcliffe (1978). Note that the motion of the user is expected to be slow, so dynamic effects, including the force of the damper, are expected to be small. According to Equation (3), if the velocity and acceleration increases, the resistance counter effect also increases. Thus, the damper is incorporated to help prevent the spring from creating a rapid return since it could be a source of risk for the user. Additionally, the damping effect limits the occurrence of high-speed movement during exercise.

\section{Resistance}

A coil spring and damper system, acting as the prismatic joint in a slider-crank mechanism, provides resistance. Using this force-generating slider-crank system in conjunction with the 4-bar linkage creates a nearly optimal resistance curve that approximates the strength curve of the user

$$
\text { Equation (4): } \frac{1}{2} I^{*}=\sum_{i=1}^{n} \frac{1}{2} m_{i}\left(\dot{x}_{i}^{2}+\dot{y}_{i}^{2}\right)+\frac{1}{2} I_{i} \dot{\theta}_{i}^{2}
$$

Equation 4 takes into account the mass $(\mathrm{m})$ and inertia $(I)$ of every moving link in the mechanism. While all links contribute to the total user force, the mass of the foot pedal is of special concern. Because the foot pedal is at the extreme end of link 3, it has the largest peak velocities and accelerations. It is also the most massive element in the prototype system. One goal in designing the device is to minimize inertial forces. This allows us to shape the static resistance curve (through kinematics) to be as similar to the human strength curve as possible. Dynamic forces will change the shape of this curve as a function of how rapidly the user moves the foot pedal. Further analysis will show that the dynamic forces can be kept small. through the range of motion. The range of motion is defined by $\theta$; from 45 degrees, the start position, to 125 degrees, the end position. This system creates the high forces and stresses needed to maintain bone density and optimize the cardiovascular workout. The slider-crank mechanism compresses the linear spring, creating an increasing resistance throughout the movement and causing the largest load to be applied when the user's leg is fully extended. This then provides the desired optimized profile in relation to the human strength curve.

\section{Resistance Due to Inertial Forces}

The user must overcome the static spring forces, the damping forces, and the inertia forces generated by acceleration of the links of the exercise device. Inertia forces are incorporated in Equation (3) by calculating an equivalent inertia of the system, $I^{*}$, that varies with position. Equation (4) from Suh and Radcliffe (1978) shows how such an equivalent inertia is found.

\section{Biomechanics}

Ground reaction forces (GRF) are created by static and dynamic loading. The forces experienced in $1 \mathrm{G}$ are due to the user's weight (static), and the dynamic loading due to movement. To simulate forces equivalent to those experienced in $1 \mathrm{G}$, the GRF must be equal to or greater than $1 \mathrm{BW}$. As shown in Equation (5), the GRF are directly related to the pressure differential force and the total user force applied by the user to move the foot pedal. Note that the vacuum feature of the LBNP Box will not be used during preliminary testing. 
Equation (5): GRF $=($ Pressure Differential Force $)+($ Total User Force $)$

Equation 5 states that the GRF during exercise in LBNP while supine and in microgravity, equals the pressure differential force plus the total user's force. The pressure differential force equals the product of the body cross-sectional area $\left(\mathrm{A}_{\mathrm{xy}}\right)$ and the pressure differential $(\Delta \mathrm{P})$ across the LBNP Box, which will be assumed to equal $50 \mathrm{mmHg}$. The total user's force includes the inertial forces caused by the geometry of the exercise portion of the multi-platform and the force required to overcome the resistance of the coil spring and damper system.

\section{A Two Member Chair Serves as Daily Activity}

The posterior side of the lower extremities are accustomed to $2 / 3 \mathrm{BW}$ between six and eight hours a day. The chair simulates this daily activity of sitting by translating a fixed linear force to the active areas. The force applied will be simulated from the negative pressure in the LBNP Box.

As shown in Equation (6), if the subject is motionless, the Total User Force term in Equation (5) equals zero.

$$
\text { Equation (6): } \mathrm{GRF}=\mathrm{A}_{\mathrm{xy}} * \Delta \mathrm{P}
$$

The chair is adjustable in both, angle and linear distance, via use of quick release pins. It is easily foldable and has a resting position horizontal to the center bar. The chair is cushioned by foam and covered with leather. The physiological and biomechanical responses of each subject will be recorded in the supine and upright position in order to collect comparative data. Methods and data results from the chair portion will be presented in future publications.

\section{Mechanical Results}

The multi-platform device was designed to accommodate the average sized astronaut, to be integrated within an existing LBNP Box, and to simulate responses found in both upright exercise and the daily activity of sitting. To collect comparable data, the multi-platform had to go from a horizontal position within the LBNP Box, to vertical position outside the LBNP Box, and allow subjects to perform the same protocol.

To accommodate a wide range of users, aside from the average astronaut, the location of the pedal system is adjustable relative to the seat location. This is accomplished through the use of a sliding member that allows the user to adjust the position of the device along a rectangular base frame. The sliding member is easily adjusted over a $14 \mathrm{~cm}$ range by a spring-loaded knob and pin detent system.

To further accommodate users of different strengths, an additional adjustment has been designed into the machine. This feature personalizes the device by changing the initial preload in the spring. The geometry of the slidercrank mechanism is changed by lowering the ground pivot on the right side of the mechanism, as shown in Figure 4. Lowering this pivot (changing the value of $l_{3}$ ) causes a vertical shift in the resistance curve, compared with a force with same magnitude caused by user's muscle strength. This can be demonstrated by Equation (7), the angular momentum of a fixed axis $\left(\mathrm{M}_{\mathrm{F}}\right)$ where $\sin \beta$ is the angle between the vectors $\left(\mathrm{F}_{\text {user }}\right)$ and $\left(l_{3}\right)$.

$$
\text { Equation (7): } \mathrm{Mf}=\mathrm{F}_{\text {user }} \mathrm{l}_{3} \sin \beta
$$

\section{The Integration of the Multi-Platform Device and the Existing LBNP Box}

The multi-platform device is manufactured to be removable, without disassembly, from the LBNP Box inner structure. It attaches to the trolley system, shown in Figure 5, making it maneuverable and easily accessible, which allows the user to adjust it to their personal settings outside of the LBNP Box. The parallel arms and seat collapse horizontally to the center bar, allowing the removal process to be quick, easy, and safe. 
The Integration of the Multi-Platform Device and the Upright Device

The physiological and biomechanical responses of each subject will be recorded in the supine and upright position in order to collect comparative data. In the upright position, there will be no added negative pressure or suction force, only the effects of gravity. Data collected in upright position will be compared to similar data taken in the supine position. If the LBNP is effective, the user's responses should be most similar between the two configurations. The design and integration of the multi-platform device in the upright position are shown in Figure 6.
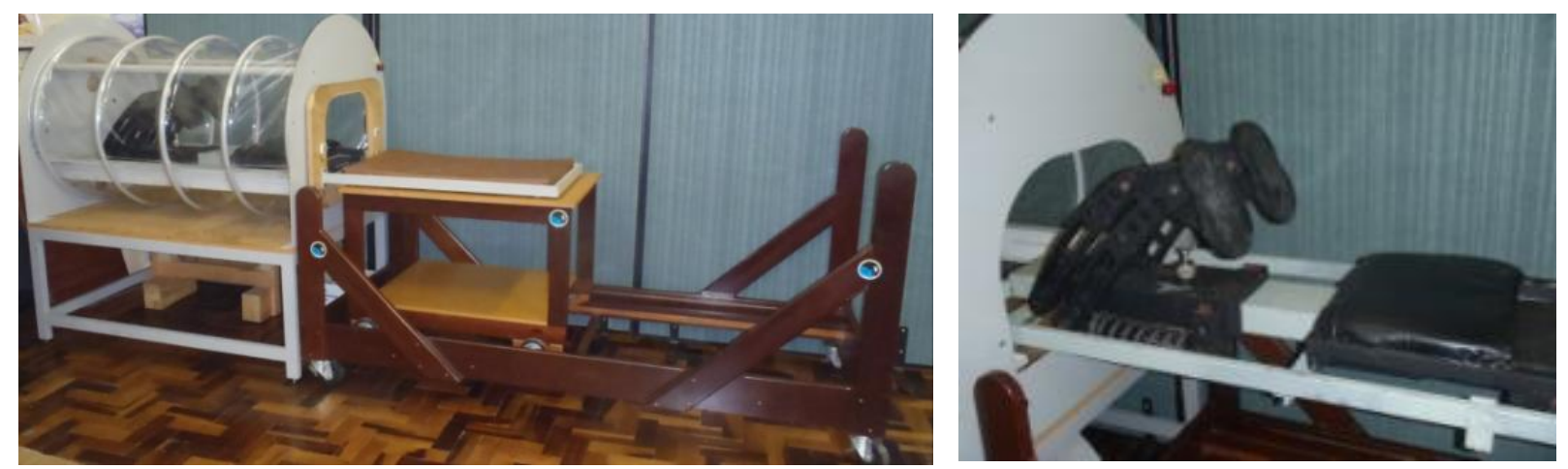

Figure 5. The left photo shows final integration of the multi-platform to the existing LBNP Box. The right photo displays a close up of the multi-platform outside of the LBNP Box.
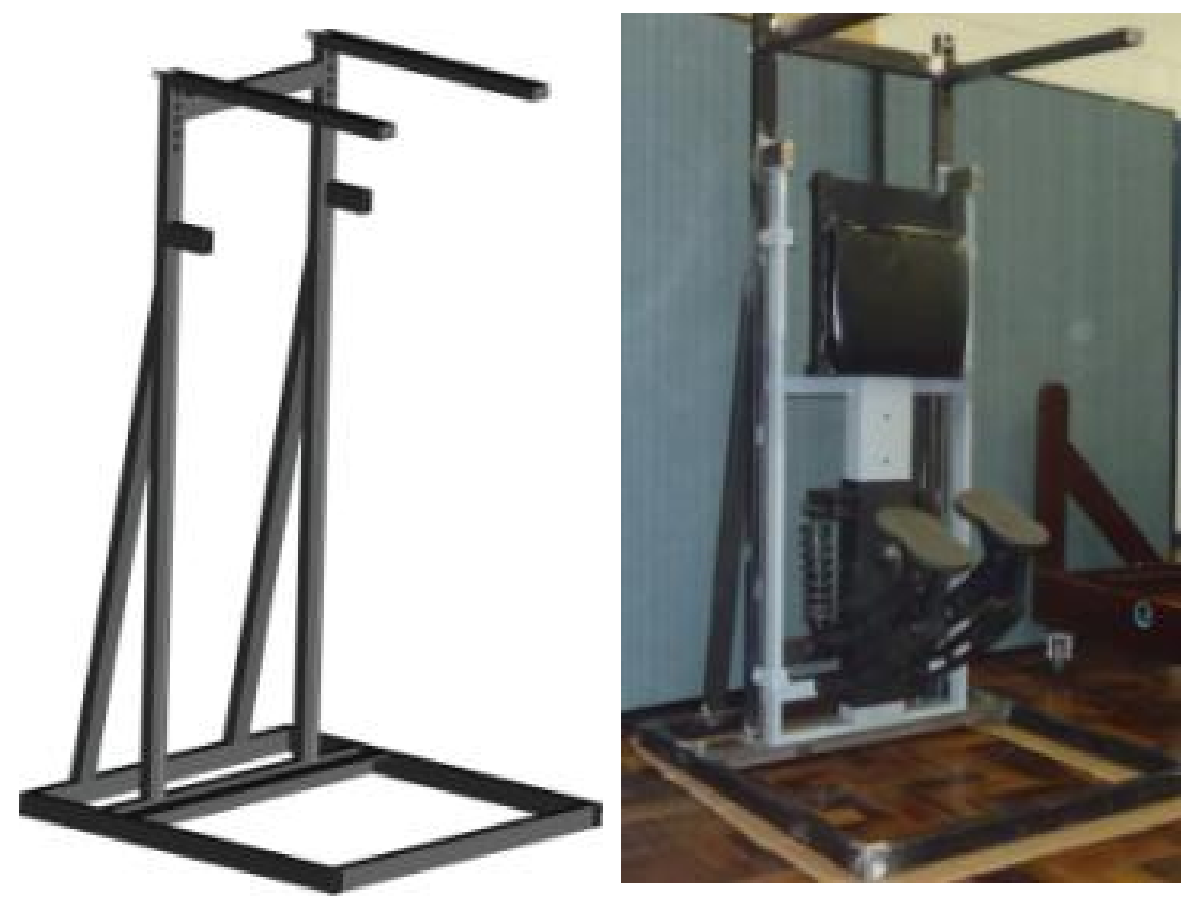

Figure 6. A 3-D CAD model, left, of the upright device that will support the multi-platform in a vertical position. The right photograph is the final integration of the multi-platform and the upright device.

\section{THEORETICAL RESULTS AND DISCUSSION}

In the next design iteration, a linear actuator will be incorporated to control the position of the above-mentioned ground pivot. The adjustment will occur automatically based on the user's heart rate (HR). The user will be required to keep a steady target HR that will be determined using Equation (8) and monitored throughout the workout. 
Equation (8): $\mathrm{HR}_{\text {target }}=\left(\left(\mathrm{HR}_{\max }-\mathrm{HR}_{\text {rest }}\right) * \%\right.$ intensity $)+\mathrm{HR}_{\text {rest }}$

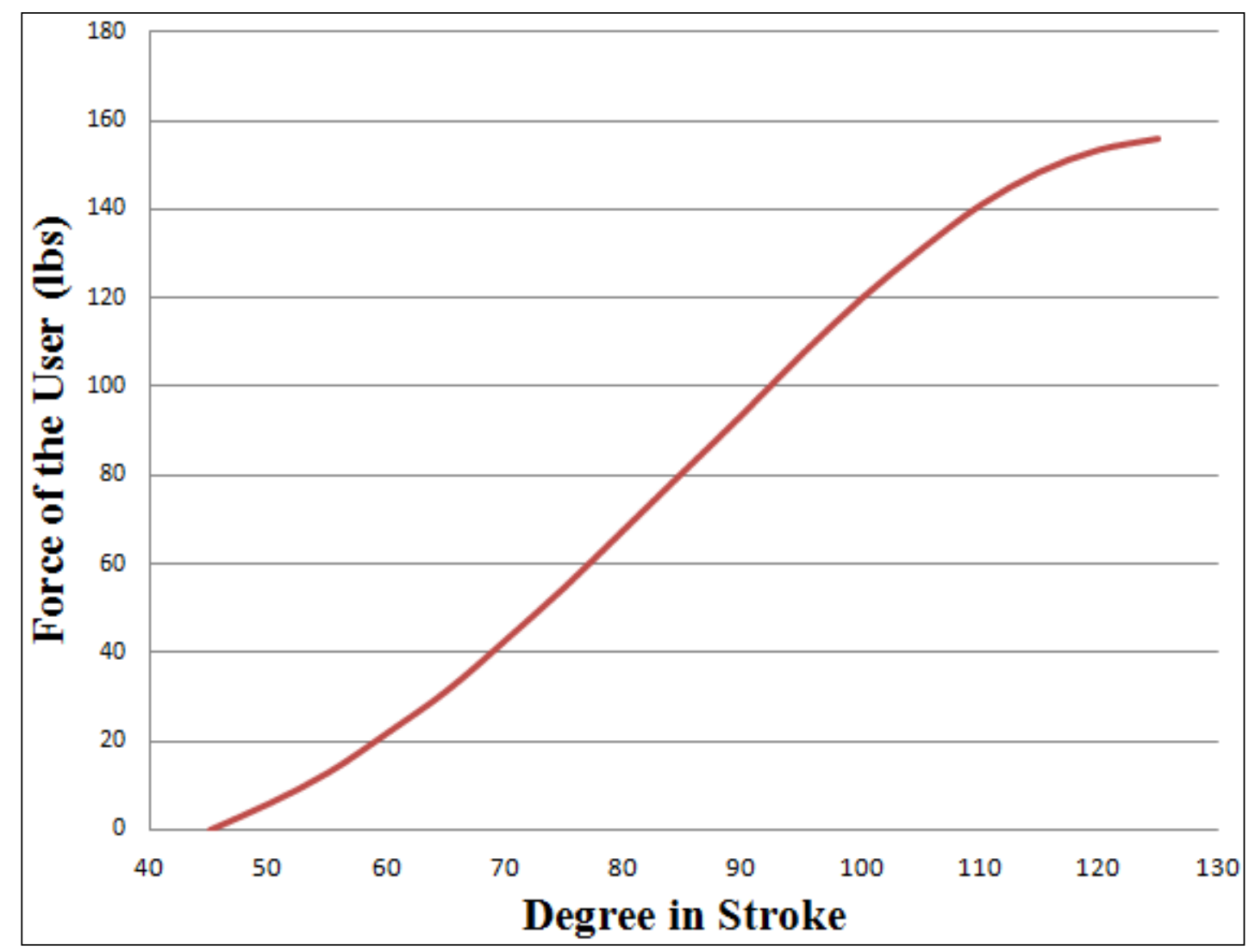

Figure 7. Theoretical static resistance curve on the outward stroke for the multi-platform considering only spring resistance.

\section{Static Resistance}

The multi-platform was to approximate the resistance provided by the machine with the human strength curve in a leg press exercise. As shown in Figure 7, the slider-crank mechanism used in the multi-platform creates a feasible approximation to the human strength curve when considering only the resistance of the spring. By limiting dynamic forces, the results show that the overall machine exhibits an increasing resistance curve under typical operating conditions.

\section{Total Theoretical Resistance}

The theoretical resistance provided by the multi-platform device has been calculated under a set of assumed conditions. This analysis uses the actual link masses and inertias from the prototype with the exception of the foot pedal's mass. In the final design, these values should be reduced. This should result in improved resistance profiles. The most important assumption necessary to perform a complete analysis is the user's motion profile. Since the foot pedals reciprocate, we know that their angular velocity will be zero at the beginning and end of each stroke. Velocity should ramp up to a peak somewhere between these endpoints. But, there is no way to precisely predict how the user will accelerate and decelerate. We do know from testing that a typical user moves at about one cycle of motion per second. The results from the two assumed motion profiles are shown in Fig. 8 and Fig. 9. In both figures, the red curve shows the user force on the foot pedal due to the resistance of the spring, the green curve shows the user force on the pedal due to dynamic effects, and the blue curve is the net user force on the pedal through a 0.5 second stroke. 


\section{Resistance Exercise Machine within LBNP Countermeasure}

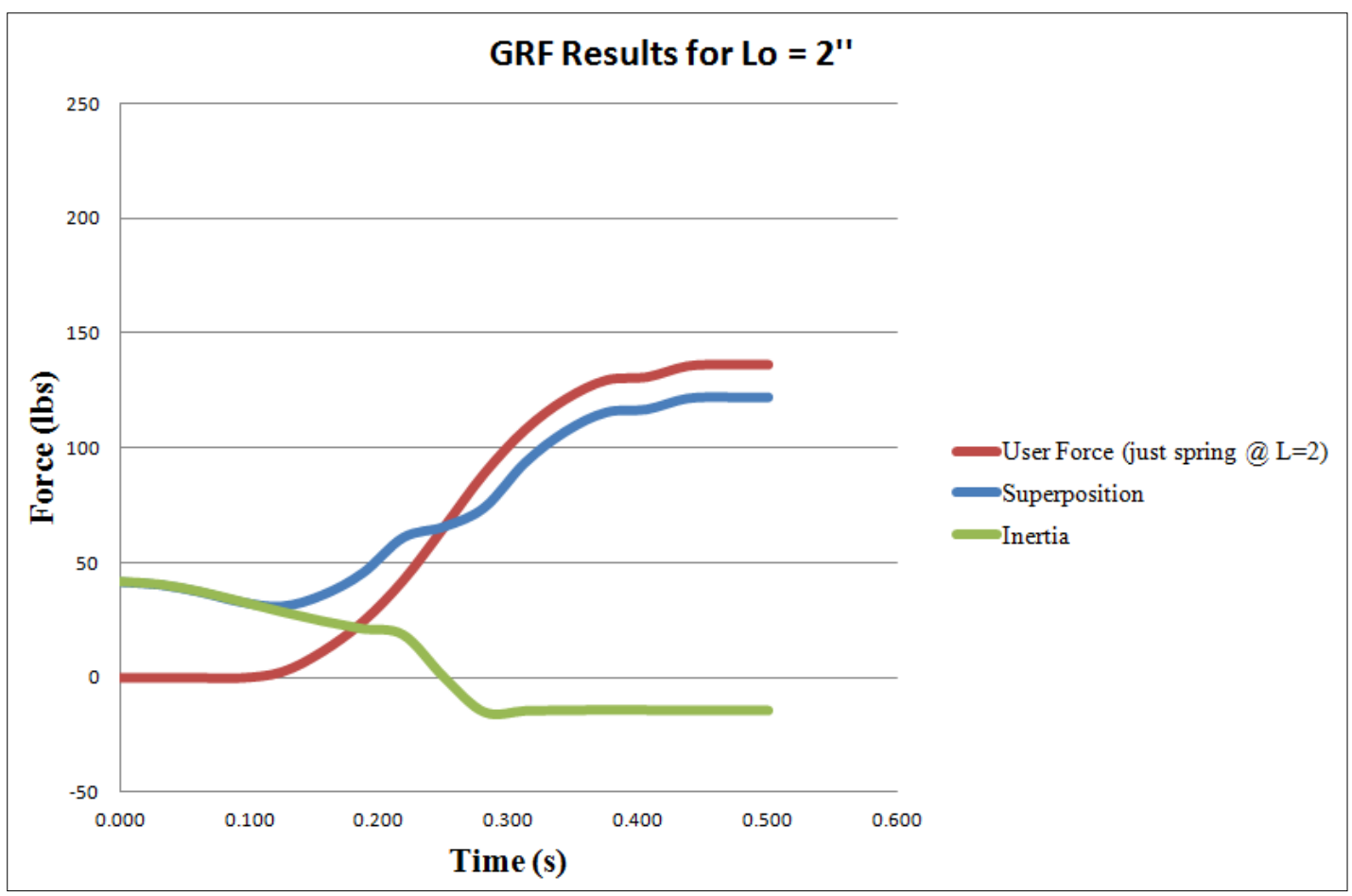

Figure 8. Theoretical resistance curve assuming a motion profile with a constant angular acceleration to start and end the motion cycle and a period of constant velocity in between. The pressure differential set at the recommended $50 \mathrm{mmHg}$.

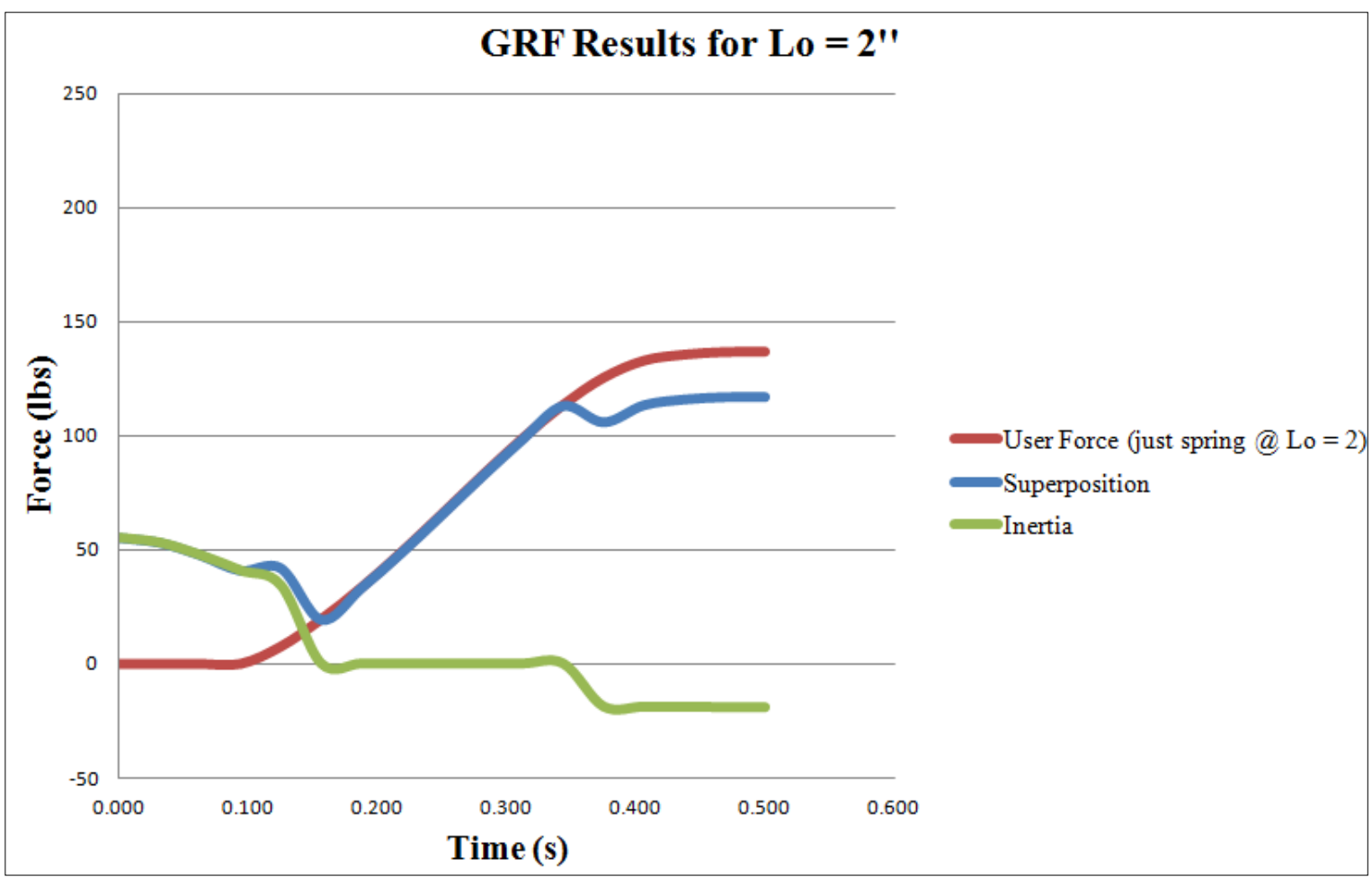

Figure 9. Theoretical resistance curve assuming a motion profile with positive and negative constant acceleration without a period of constant velocity. The pressure differential is set at $\mathbf{- 5 0} \mathbf{m m H g}$. 
The output data from the electrogoniometer indicated that the user is generally accelerating or decelerating the foot pedal, with little or no constant velocity in the middle. As a result, the second velocity profile will be assumed for all subsequent analysis.

The analysis also considered the effect of varying the spring preload and the effect of the LBNP Box pressure difference on the foot pedal forces exerted by the user. The graphs in Fig. 10 show the variation in user foot pedal force as the spring preload increases through a change in the adjustable dimension $l_{\mathrm{o}}$.

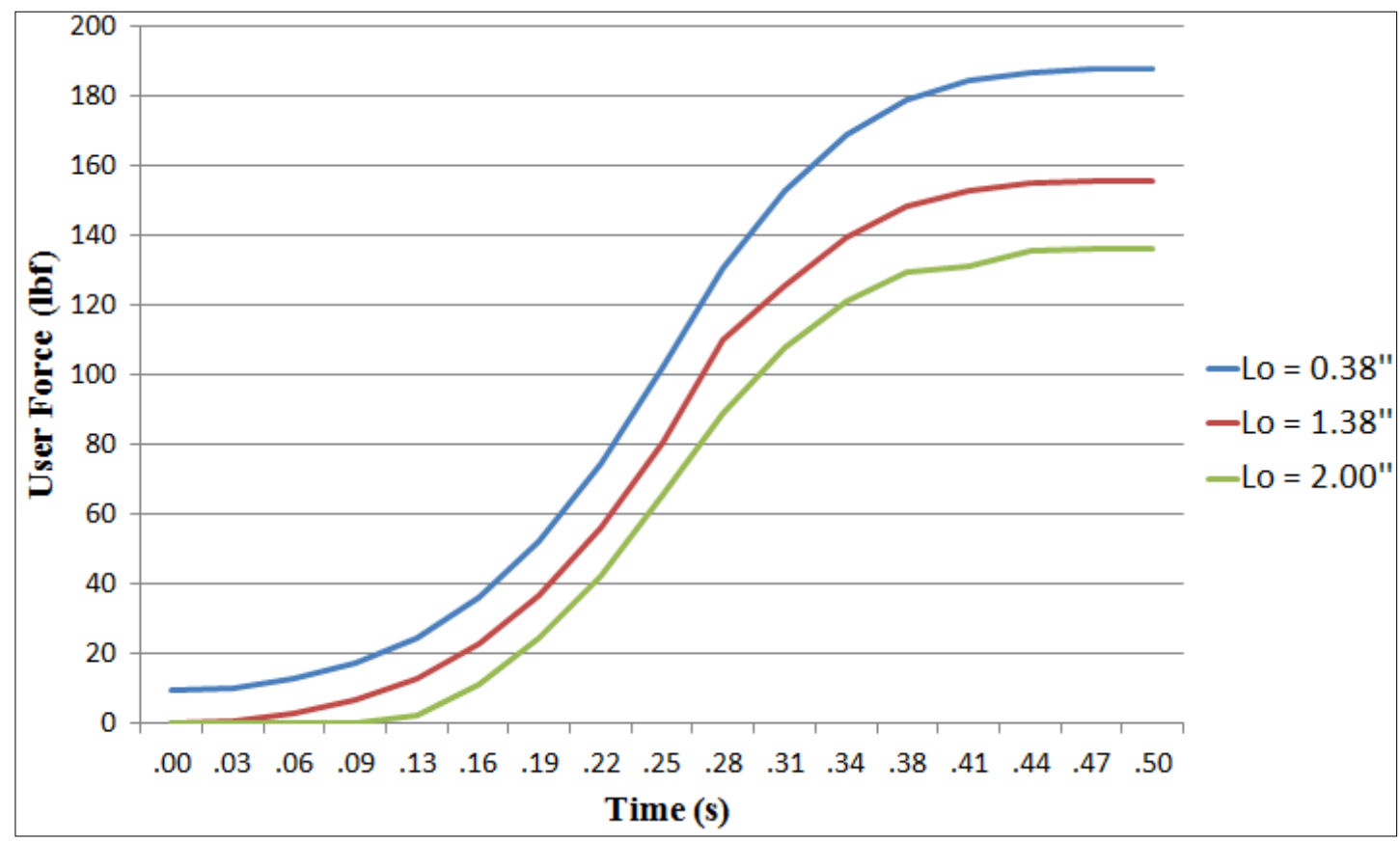

Figure 10. Variation in the user's force as the spring preload increases through a change in dimension $l_{0}$.

\section{SUMMARIES AND CONCLUSION}

The compact, easily transportable, multiplatform device is designed to simulate both exercise and the daily activity of sitting. The exercise portion of the device creates stress on the lower extremities by supplying a variable resistance to a reciprocating foot pedal. This resistance is created from a coil spring and damper system acting through a 4-bar linkage. The resisting force increases as a function of leg extension to maximize work done by the user in each cycle of motion. The sitting portion of the multi-platform device creates a resistance applied to the posterior side of the lower extremities by the use of a chair. The chair is adjustable in angle to fit each subject and to simulate a force $2 / 3 \mathrm{BW}$, mimicking the posterior forces equivalent to the human activity of sitting between 6-8 hours a day.

The multi-platform is paired with an existing LBNP Box to add an evenly distributed pressureinduced stress to the lower extremities. However, the LBNP Box constrains the length of the subject's lower extremities, waist to sole of foot, to range from $70 \mathrm{~cm}$ to $82 \mathrm{~cm}$. By combining resistance exercise and lower body negative pressure, the subject will experience one or more times BW in stress on their musculoskeletal, cardiovascular and nervous systems. By achieving $1 \mathrm{BW}$ or greater (artificial gravity) during exercise and $2 / 3$ BW during sitting, the gap between the precondition and post condition syndrome will become smaller. The largest single-leg forces during resistance exercise are $1.16 \mathrm{BW}(232 \mathrm{lbs})$ during supine position when $\gamma$, the angle between the horizontal and the ground pivot on the right side of the mechanism, equals 187 degrees and minimal at $0.68 \mathrm{BW}(136 \mathrm{lbs})$ when $\gamma$ equals 177 degrees. We conclude that the exercise portion of the multi-platform was able to elicit loads comparable to exercise on Earth since the forces were greater than $1 \mathrm{BW}$ and predict that when paired with LBNP the maximum resistance load can be as low as $196 \mathrm{lbf}$ when the LBNP is set for 
the recommended $50 \mathrm{mmHg}$ to achieve, at maximum, $2 \mathrm{BW}$.

Future versions of the machine should have lighter links and hence improved overall resistance curves. The multi-platform is fabricated from steel, which causes the inertia forces in the above calculations to be larger than desired. The angle of the foot pedal needs to be adjusted so that the user's foot maintains an angle closer to $90^{\circ}$ throughout the entire cycle rather than just toward the beginning and the end of the stroke. Currently, too much of the force from the subject's foot is directed along the link, resulting in user forces that are somewhat higher than desired for the first half of the pedal stroke. Another future improvement includes a linear actuator to change the level of resistance based directly off the subject's heart rate.
Overall, the combination of the multiplatform and the LBNP Box show great promise for minimizing deconditioning and for providing a safe, compact, lightweight and efficient way for space travelers to exercise.

\section{ACKNOWLEDGEMENTS}

This study was conducted under the partnership of Embry-Riddle Aeronautical University, USA and Pontifícia Universidade do Rio Grande do Sul, Brazil and was supported by Fund for the Improvement of Postsecondary Education (FIPSE) and Fundação de Amparo à Pesquisa do Estado do Rio Grande do Sul (FAPERGS), Brazil. The authors also thank the Office of Naval Research for support of this research.

\section{Nomenclature}

$\begin{array}{ll}\text { BW } & \text { Body weight } \\ \mathrm{G} & \text { Gravity } \\ \mathrm{GRF} & \text { Ground reaction force } \\ \mathrm{HR} & \text { Heart rate } \\ \text { LBNP } & \text { Lower body negative pressure } \\ \gamma & \text { The angle between the horizontal and the ground pivot on the right side of the mechanism } \\ \theta & \text { Input position } \\ \dot{\theta} & \text { Velocity } \\ \ddot{\theta} & \text { Acceleration } \\ \mathrm{S} & \text { Position of the spring } \\ \dot{S} & \text { Velocity of the spring } \\ \mathrm{I}^{*} & \text { Equivalent inertia of the system } \\ I & \text { mass of inertia } \\ \mathrm{m} & \text { mass } \\ F_{\text {spring }} & \text { Resistance provided by the spring } \\ F_{\text {user }} & \text { Resistive force } \\ l_{0} & \text { Link length of the member between the horizontal and the ground pivot on the right side of } \\ & \text { the mechanism } \\ l_{1} & \text { Link length of the member between } l_{o} \text { and the rotational pivot } \\ l_{2} & \text { Link length of the member below the rotational pivot } \\ l_{3} & \text { Link length of the member above the rotational pivot } \\ \Delta \mathrm{P} & \text { Pressure differential force- the difference between the pressures inside the Box vs. outside } \\ & \text { the Box } \\ \mathrm{A}_{\mathrm{xy}} & \text { Cross sectional area of the seal which is placed around the subject's waist } \\ \beta & \text { The angle between vector } F_{\text {user }} \text { and the arm } l_{3}\end{array}$

\section{REFERENCES}

Baqai FP, Gridley DS, Slater JM, Luo-Owen X, Stodieck LS, Ferguson V, Chapes SK, Pecaut MJ (2009) Effects of spaceflight on innate immune function and antioxidant gene expression. Journal of Applied Physiology 106(6): 1935-1942

Benson, A (2002) Motion sickness. In Medical Aspects of Harsh Environments textbooks of military medicine, K.B. Pandoff, R.E. Burr 
(eds),Washington, DC: Office of the Surgeon General, Department of the Army, Volume 2, pp 1049-1055

Berg HE, Eiken O, Miklavcic L, Mekjavic IB (2007) Hip, thigh, and calf muscle atrophy and bone loss after 5 -week bedrest inactivity. European Journal of Applied Physiology 99: 283-289

Boda WL, Watenpaugh DE, Ballard RE, Hargens AR (2000) Supine lower body negative pressure exercise simulates metabolic and kinetic features of upright exercise. Journal of Applied Physiology 89: 649-654

Caillot-Augusseau A, Lafage-Proust M-H, Soler C, Pernod J, Dubois F, Alexandre C (1998) Bone formation and resorption biological markers in cosmonauts during and after a 180-day spaceflight (Euromir 95). Clinical Chemistry 44(3): 578-585

Charles JB, Lathers CM (1991) Cardiovascular adaptation to spaceflight. Journal of Clinical Pharmacology 31(1): 1010-1023

Convertino VA, Cooke WH (2005) Evaluation of cardiovascular risks of spaceflight does not support the NASA bioastronautics critical path roadmap. Aviation, Space, and Environmental Medicine 76: 869-876

Davis BL, Cavanaugh PR (1993) Simulating reduced gravity: a review of biomechanical issues pertaining to human motion. Aviation, Space, and Environmental Medicine 64(6): 557-566

Dorfman TA, Levine BD, Tillery T, Peshock RM, Hastings JL, Schneider SM, Macias BR, Biolo G, Hargens AR (2007) Cardiac atrophy in women following bed rest. Journal of Applied Physiology 103: 8-16

Gazenko OG, Gurovsky NN, Genin AM, Bryanov II, Eryomin AV, Egorov AD (1976) Results of medical investigations carried out on board the Salyut orbital stations. Life Science 14: 145-152

Gianoudis J, Bailey CA, Sanders KM, Nowson CA, Hill K, Ebeling PR, Daly RM (2012) Osteo-cise: strong bones for life: protocol for a community-based randomised controlled trial of a multi-modal exercise and osteoporosis education program for older adults at risk of falls and fractures. BMC Musculoskeletal Disorders. 28:13: 78

Hughson RL,Shoemaker JK, Blaber AP, Arbeille $\mathrm{P}$, Greaves DK, Pereira-Junior PP, Xu D (2012) Cardiovascular regulation during long-duration spaceflights to the International Space Station. Journal of Applied Physiology 112: 719-727

Lang T, LeBlanc A, Evans H, Lu Y, Genant H, Yu A (2004) Cortical and trabecular bone mineral loss from the spine and hip in longduration spaceflight. Journal of Bone and Mineral Research 19: 1006-1012

Martinelli L, Russomano T, Santos M, Falcao F, Bauer M, Machado A, Sundaresan A (2009) Effect of microgravity on immune cell viability and proliferation simulation using 3-D clinostat. IEEE Engineering in Medicine and Biology Magazine 28(4): 85-90

Morgan JL, Zwart SR, Heer M, Ploutz-Snyder R, Ericson K, Smith SM (2012) Bone metabolism and nutritional status during 30day head-down-tilt bed rest. Journal of Applied Physiology 113(10): 1519-1529

Mulavara AP, Feiveson AH, Fiedler J, Cohen H, Peters BT, Miller C, Brady R, Bloomberg JJ (2010) Locomotor function after longduration spaceflight: effects and motor learning during recovery. Experimental Brain Research 202: 649-659

NASA (2000) Anthropometric Source Book: Volume 1: Anthropometry for Designers Anthropology Staff/Webb Associates, NASA RP 1024, pp 7-78

Russomano T, Falcão F, Gurgel J, Piccoli L, Porto F, Dalmarco G, de Azevedo DFG, Allan J, Beck L, Petrat G, Thompsen S (2005) Development of a lower body negative pressure box with an environmental control system for physiological studies. Proceedings of the Annual International Conference of the IEEE Engineering in Medicine and Biology Society 5: 4501-4504

Schulz BW, Hart-Hughes S, Gordon MT, Bulat T (2012) Training implications of maximal forces on a computer-controlled and motordriven leg press by age group, sex, footplate direction, and speed. Experimental Gerontology 47: 295-303

Shackelford LC (2008) Musculoskeletal response to spaceflight, In Principles of Clinical Medicine for Spaceflight, M.R. Barratt, S.L. Pool (eds), pp 293-306. New York: Springer

Shackelford LC, LeBlanc AD, Driscoll TB, Evans HJ, Rianon NJ, Smith SM, Spector E, Feeback DL, Lai D (2004) Resistance exercise as a countermeasure to disuseinduced bone loss. Journal of Applied 
Physiology 97: 119-129

Smith SM, Nillen JL, LeBlanc A, Lipton A, Demers LM, Lane HW, Leach CS (1998) Collagen cross-link excretion during spaceflight and bed rest. Journal of Clinical Endocrinology and Metabolism 83(10): 3584-3591

Suh CH, Radcliffe CW (1978) Kinematics and Mechanisms Design, New York: Wiley

Thornton WE, Hoffler GW, Rummel JA (1977) Anthropometric changes and fluids shifts, In Biomedical Results from Skylab. RS Johnston, LF Dietlein, (eds.), pp 330-338. Washington, DC : Scientific and Technical Information Office, National Aeronautics and Space Administration. NASA SP-377

Tidwell PH, Soper RR, Reinholtz CF (1996) Synthesis of Force-Generating Mechanisms, Proceedings of the 24th Biennial Mechanisms Conference, Irvine, California, August 18-22, paper 96-DETC/MECH-1211

Trappe S, Costill D, Gallagher P, Creer A, Peters JR, Evans H, Riley D, Fitts R (2009) Exercise in space: human skeletal muscle after 6 months aboard the International Space Station. Journal of Applied Physiology 106(4): $1159-1168$
Vorobyev YI, Gazenko OG, Gurovskiy NN, Nefedov YG, Yegorov BB, Bayevskiy RM, Bryanov II, Genin AM, Degtyarev VA, Yegorov AD, Yeremin AV, Pestov ID (1976) Preliminary results of medical investigations carried out during flight of the second expedition of the Salyut-4 orbital station. Kosmicheskaya Biologiya I Aviakosmicheskaya Meditsina 10(5): 3-18

Watenpaugh DE (2001) Fluid volume control during short-term spaceflight and implications for human performance. The Journal of Experimental Biology 204: 32093215

Watenpaugh DE, Hargens AR (1996) The cardiovascular system in microgravity. In Handbook of Physiology: Environmental Physiology, Vol 1, Bethesda, MD: American Physiological Society, Sect 4, Vol 1, 29: 631-674

Zwart SR, Hargens AR, Lee SMC, Macias BR, Watenpaugh DE, Tse K, Smith SM (2007) Lower body negative pressure treadmill exercise as a countermeasure for bed restinduced bone loss in female identical twins. Bone 40(2): 529-537 